\title{
Improving the Accuracy of the Linear Interacion Energy Method for Solvation Free Energies
}

\author{
Martin Almlöf ${ }^{\dagger}$, Jens Carlsson ${ }^{\dagger}$, Johan Åqvist* \\ Department of Cell and Molecular Biology, Uppsala University, Biomedical Center, Box 596,
}

SE-751 24 Uppsala, Sweden

*Corresponding author: Phone: +46 18471 4109, Fax: +46 185369 71, E-mail: aqvist@xray.bmc.uu.se

${ }^{\dagger}$ These authors contributed equally to this work.

Keywords: solvation free energies, molecular dynamics, linear interaction energy, linear response

Abbreviations: MD, molecular dynamics; FEP, free energy perturbation; rms, root mean square 
Table 1. The experimental hydration free energies $\left(\Delta F_{\text {sol }}^{\text {obs }}\right)(\mathrm{kcal} / \mathrm{mol})$, electrostatic solute energies $\left(\left\langle U_{r-s}^{e l}\right\rangle_{B}^{w}\right.$ and $\left.\Delta\left\langle U_{r-r}^{e l}\right\rangle_{B}\right)(\mathrm{kcal} / \mathrm{mol})$, calculated values of $\beta_{F E P}$, electrostatic contribution to the free energies of hydration calculated using FEP $\left(\Delta F_{\text {sol }}^{e l}(F E P)\right)$ and Model D/E $\left(\Delta F_{\text {sol }}^{e l}(\right.$ Model $\left.D / E)\right)$ $(\mathrm{kcal} / \mathrm{mol})$ for each of the molecules in the training set.

\begin{tabular}{|c|c|c|c|c|c|c|c|}
\hline Solute & $\Delta F_{\text {sol }}^{\text {obs a,b }}$ & $\left\langle U_{r-s}^{e l}\right\rangle_{B}^{w}$ a,c & $\Delta\left\langle U_{r-r}^{e l}\right\rangle_{B}$ a,d & $\left\langle U_{r-s}^{v d W}\right\rangle_{B}^{w}$ a,e & $\beta_{F E P}$ & $\Delta F_{\text {sol }}^{e l}(F E P)^{\mathrm{a}, \mathrm{f}}$ & $\Delta F_{\text {sol }}^{e l}(\text { Model } D / E)^{\mathrm{a}}$ \\
\hline Ethane & 1.83 & -0.06 & 0.01 & -5.38 & -0.10 & 0.01 & -0.02 \\
\hline Propane & 1.96 & -0.07 & 0.00 & -7.29 & 0.00 & 0.00 & -0.03 \\
\hline Butane & 2.08 & -0.09 & 0.19 & -9.06 & 1.10 & 0.11 & 0.04 \\
\hline Isobutane & 2.32 & -0.06 & 0.01 & -8.88 & -0.22 & 0.01 & -0.02 \\
\hline Pentane & 2.33 & -0.12 & -0.05 & -10.79 & -0.44 & 0.07 & -0.07 \\
\hline 2-Methylbutane & 2.38 & -0.08 & 0.24 & -10.49 & 1.09 & 0.18 & 0.07 \\
\hline Neopentane & 2.50 & -0.05 & 0.00 & -10.42 & -0.22 & 0.01 & -0.02 \\
\hline Hexane & 2.49 & -0.13 & 0.29 & -12.53 & 1.88 & 0.30 & 0.07 \\
\hline 2-Methylpentane & 2.52 & -0.11 & 0.18 & -12.29 & 0.31 & 0.02 & 0.03 \\
\hline 3-Methylpentane & 2.51 & -0.11 & -0.02 & -12.22 & 1.31 & -0.17 & -0.06 \\
\hline 2,3-Dimethylbutane & 2.59 & -0.07 & 0.03 & -12.00 & -1.13 & 0.04 & -0.02 \\
\hline Cyclopropane & 0.75 & -0.12 & 0.00 & -6.50 & 0.35 & -0.04 & -0.05 \\
\hline Cyclopentane & 1.20 & -0.14 & 0.02 & -9.92 & 0.04 & -0.01 & -0.05 \\
\hline Methylcyclopentane & 1.60 & -0.12 & -0.07 & -11.56 & 0.41 & -0.08 & -0.08 \\
\hline Cyclohexane & 1.23 & -0.06 & 0.00 & -11.60 & 0.00 & 0.00 & -0.03 \\
\hline Methylcyclohexane & 1.71 & -0.06 & 0.02 & -12.97 & -0.44 & 0.02 & -0.02 \\
\hline Methane & 1.91 & -0.03 & 0.00 & -3.27 & 0.00 & 0.00 & -0.01 \\
\hline Heptane & 2.62 & -0.15 & 0.20 & -14.26 & 2.30 & 0.12 & 0.02 \\
\hline Octane & 2.89 & -0.16 & -0.04 & -15.94 & 0.83 & -0.17 & -0.09 \\
\hline Ethylene & 1.27 & -0.81 & 0.02 & -4.63 & 0.40 & -0.32 & -0.34 \\
\hline 1-Propene & 1.27 & -0.85 & 0.00 & -6.63 & 0.41 & -0.35 & -0.37 \\
\hline 1-Butene & 1.38 & -0.91 & 0.13 & -8.43 & 0.29 & -0.23 & -0.34 \\
\hline 2-Methyl-2-butene & 1.31 & -0.71 & -0.01 & -10.31 & 0.41 & -0.30 & -0.31 \\
\hline 1-Hexene & 1.68 & -0.91 & 0.09 & -11.86 & 0.26 & -0.21 & -0.35 \\
\hline trans-2-Pentene & 1.34 & -0.88 & -0.13 & -10.39 & 0.36 & -0.36 & -0.43 \\
\hline 2-Methyl-1,3-butadiene & 0.68 & -2.74 & 0.33 & -9.60 & 0.35 & -0.85 & -1.04 \\
\hline 1,4-Pentadiene & 0.94 & -1.62 & 0.24 & -9.65 & 0.30 & -0.42 & -0.59 \\
\hline Cyclopentene & 0.56 & -1.12 & 0.00 & -9.52 & 0.39 & -0.43 & -0.48 \\
\hline Butadiene & 0.60 & -1.90 & -0.03 & -7.86 & 0.44 & -0.86 & -0.83 \\
\hline 1-Pentene & 1.40 & -0.84 & 0.09 & -10.09 & 0.39 & -0.30 & -0.32 \\
\hline Acetylene & -0.01 & -2.69 & 0.00 & -3.02 & 0.44 & -1.17 & -1.16 \\
\hline 1-Propyne & -0.31 & -1.34 & 0.00 & -6.00 & 0.48 & -0.64 & -0.57 \\
\hline 1-Butyne & -0.16 & -1.43 & 0.00 & -8.00 & 0.46 & -0.65 & -0.61 \\
\hline 1-Pentyne & 0.01 & -1.37 & -0.02 & -9.84 & 0.47 & -0.65 & -0.60 \\
\hline 1-Hexyne & 0.29 & -1.47 & 0.16 & -11.55 & 0.34 & -0.45 & -0.56 \\
\hline 1-Buten-3-yne & 0.04 & -2.29 & 0.00 & -7.12 & 0.45 & -1.03 & -0.98 \\
\hline Benzene & -0.87 & -4.51 & 0.01 & -9.72 & 0.41 & -1.85 & -1.94 \\
\hline Toluene & -0.89 & -6.33 & 0.00 & -11.30 & 0.41 & -2.57 & -2.72 \\
\hline o-Xylene & -0.90 & -7.76 & 0.00 & -12.96 & 0.44 & -3.45 & -3.34 \\
\hline m-Xylene & -0.84 & -7.89 & 0.00 & -13.00 & 0.42 & -3.30 & -3.39 \\
\hline p-Xylene & -0.81 & -7.67 & 0.00 & -13.01 & 0.42 & -3.24 & -3.30 \\
\hline Ethylbenzene & -0.80 & -5.95 & 0.01 & -12.98 & 0.42 & -2.50 & -2.55 \\
\hline 1,2,4-Trimethylbenzene & -0.86 & -9.38 & 0.00 & -14.43 & 0.44 & -4.12 & -4.03 \\
\hline Biphenyl & -2.64 & -7.15 & 0.08 & -17.39 & 0.44 & -3.14 & -3.04 \\
\hline Naphthalene & -2.39 & -6.12 & 0.00 & -14.79 & 0.46 & -2.81 & -2.63 \\
\hline 1-Methylnaphthalene & -2.37 & -7.83 & 0.01 & -16.26 & 0.44 & -3.45 & -3.36 \\
\hline 1,3-Dimethylnaphthalene & -2.47 & -9.29 & 0.01 & -17.85 & 0.45 & -4.14 & -3.99 \\
\hline 1,4-Dimethylnaphthalene & -2.82 & -9.23 & 0.02 & -17.70 & 0.47 & -4.31 & -3.96 \\
\hline 2,3-Dimethylnaphthalene & -2.78 & -9.33 & 0.00 & -17.86 & 0.45 & -4.20 & -4.01 \\
\hline 2,7-Dimethylnaphthalene & -2.63 & -9.04 & 0.00 & -18.00 & 0.44 & -4.00 & -3.89 \\
\hline
\end{tabular}




\begin{tabular}{|c|c|c|c|c|c|c|c|}
\hline Fluorene & -3.44 & -6.00 & 0.01 & -18.52 & 0.45 & -2.68 & -2.58 \\
\hline Phenanthrene & -3.95 & -7.81 & -0.01 & -19.55 & 0.46 & -3.59 & -3.36 \\
\hline Pyrene & -4.46 & -8.16 & 0.00 & -21.40 & 0.46 & -3.78 & -3.51 \\
\hline Acenaphthalene & -3.15 & -6.03 & 0.00 & -16.73 & 0.44 & -2.68 & -2.59 \\
\hline Anthracene & -4.23 & -7.97 & 0.00 & -19.65 & 0.45 & -3.60 & -3.43 \\
\hline Isopropylbenzene & -0.30 & -6.10 & 0.00 & -14.51 & 0.43 & -2.62 & -2.62 \\
\hline t-Butylbenzene & -0.44 & -6.41 & 0.00 & -15.85 & 0.43 & -2.75 & -2.76 \\
\hline Methanol & -5.11 & -16.93 & 0.03 & -1.45 & 0.38 & -6.50 & -6.30 \\
\hline Ethanol & -5.01 & -17.72 & -0.18 & -3.39 & 0.38 & -6.87 & -6.67 \\
\hline 1-Propanol & -4.83 & -17.28 & 0.41 & -5.22 & 0.36 & -6.10 & -6.29 \\
\hline 2-Propanol & -4.76 & -17.89 & -0.15 & -5.12 & 0.37 & -6.63 & -6.73 \\
\hline 1-Butanol & -4.72 & -16.87 & 0.42 & -7.18 & 0.37 & -6.15 & -6.13 \\
\hline 2-Methyl-1-propanol & -4.52 & -16.54 & 0.37 & -7.08 & 0.37 & -5.99 & -6.03 \\
\hline 2-Butanol & -4.58 & -17.03 & 0.45 & -7.09 & 0.33 & -5.52 & -6.18 \\
\hline t-Butanol & -4.51 & -17.24 & 0.07 & -6.86 & 0.38 & -6.46 & -6.40 \\
\hline 1-Pentanol & -4.47 & -17.22 & 0.06 & -8.79 & 0.37 & -6.33 & -6.40 \\
\hline 3-Methyl-1-butanol & -4.42 & -17.33 & 0.10 & -8.56 & 0.37 & -6.37 & -6.43 \\
\hline 2-Pentanol & -4.39 & -17.32 & -0.06 & -8.80 & 0.36 & -6.22 & -6.48 \\
\hline 3-Pentanol & -4.35 & -16.36 & 0.31 & -8.90 & 0.35 & -5.68 & -5.99 \\
\hline 2-Methyl-2-butanol & -4.43 & -16.79 & 0.84 & -8.63 & 0.34 & -5.49 & -5.95 \\
\hline 1-Hexanol & -4.36 & -17.22 & -0.02 & -10.57 & 0.36 & -6.27 & -6.43 \\
\hline 2,3-Dimethyl-2-butanol & -3.91 & -15.43 & -0.80 & -10.54 & 0.41 & -6.62 & -6.05 \\
\hline 3-Hexanol & -4.08 & -16.33 & -0.34 & -10.67 & 0.38 & -6.38 & -6.22 \\
\hline 4-Methyl-2-pentanol & -3.74 & -16.66 & -0.19 & -10.40 & 0.39 & -6.50 & -6.28 \\
\hline 2-Methyl-3-pentanol & -3.89 & -16.07 & 0.23 & -10.52 & 0.38 & -6.01 & -5.91 \\
\hline 2-Methyl-2-pentanol & -3.93 & -16.36 & 0.36 & -10.41 & 0.38 & -6.03 & -5.97 \\
\hline Cyclopentanol & -5.49 & -16.96 & 0.07 & -8.09 & 0.36 & -6.03 & -6.30 \\
\hline Cyclohexanol & -5.48 & -15.91 & 0.79 & -9.79 & 0.37 & -5.54 & -5.64 \\
\hline Phenol & -6.62 & -17.88 & -0.05 & -8.00 & 0.38 & -6.89 & -6.68 \\
\hline 2-Methylphenol & -5.87 & -17.61 & -0.09 & -9.95 & 0.39 & -6.89 & -6.60 \\
\hline 4-Methylphenol & -6.14 & -18.91 & -0.04 & -9.67 & 0.39 & -7.33 & -7.07 \\
\hline 4-t-Butylphenol & -5.92 & -19.39 & -0.04 & -14.00 & 0.38 & -7.39 & -7.24 \\
\hline 2-Propene-1-ol & -4.80 & -17.84 & -1.11 & -4.77 & 0.38 & -7.15 & -7.06 \\
\hline Dimethyl ether & -1.90 & -6.83 & 0.00 & -5.65 & 0.40 & -2.70 & -2.93 \\
\hline Diethyl ether & -1.63 & -7.60 & -0.04 & -9.49 & 0.40 & -3.07 & -3.29 \\
\hline Methyl n-propyl ether & -1.66 & -7.27 & 0.19 & -9.32 & 0.32 & -2.27 & -3.04 \\
\hline Methyl isopropyl ether & -2.01 & -7.41 & 0.01 & -9.11 & 0.40 & -2.93 & -3.18 \\
\hline Ethyl n-propyl ether & -1.81 & -7.32 & 0.21 & -11.09 & 0.36 & -2.58 & -3.06 \\
\hline Methyl t-butyl ether & -2.21 & -7.63 & 0.02 & -10.53 & 0.39 & -2.98 & -3.27 \\
\hline Di-n-propyl ether & -1.15 & -6.87 & -0.21 & -12.76 & 0.37 & -2.62 & -3.04 \\
\hline Di-isopropylether & -0.53 & -7.58 & -0.16 & -12.40 & 0.44 & -3.41 & -3.33 \\
\hline Di-n-butyl ether & -0.83 & -6.67 & -0.08 & -16.39 & 0.38 & -2.59 & -2.90 \\
\hline Tetrahydrofuran & -3.47 & -7.48 & 0.02 & -8.45 & 0.40 & -2.97 & -3.21 \\
\hline 2-Methyltetrahydrofuran & -3.30 & -7.97 & 0.02 & -10.10 & 0.40 & -3.19 & -3.42 \\
\hline 2,5-Dimethyltetrahydrofuran & -2.92 & -8.39 & 0.02 & -11.78 & 0.41 & -3.41 & -3.60 \\
\hline Phenyl methyl ether & -1.04 & -6.32 & -0.05 & -12.43 & 0.43 & -2.71 & -2.74 \\
\hline Tetrahydropyran & -3.12 & -7.54 & 0.02 & -10.06 & 0.37 & -2.82 & -3.23 \\
\hline Acetone & -3.85 & -10.41 & 0.00 & -6.62 & 0.43 & -4.44 & -4.48 \\
\hline 2-Butanone & -3.64 & -10.66 & 0.00 & -8.43 & 0.42 & -4.50 & -4.58 \\
\hline 2-Pentanone & -3.53 & -10.37 & 0.00 & -10.24 & 0.41 & -4.21 & -4.46 \\
\hline 3-Pentanone & -3.41 & -10.91 & 0.11 & -10.32 & 0.42 & -4.57 & -4.65 \\
\hline 3-Methyl-2-butanone & -3.24 & -10.48 & 0.00 & -10.10 & 0.43 & -4.46 & -4.51 \\
\hline 2-Hexanone & -3.29 & -10.64 & -0.41 & -11.91 & 0.44 & -4.87 & -4.75 \\
\hline 4-Methyl-2-pentanone & -3.06 & -10.37 & -0.30 & -11.70 & 0.42 & -4.49 & -4.58 \\
\hline 2-Heptanone & -3.04 & -10.54 & -0.27 & -13.51 & 0.44 & -4.80 & -4.65 \\
\hline 4-Heptanone & -2.93 & -10.65 & -0.27 & -13.71 & 0.44 & -4.79 & -4.69 \\
\hline 2,4-Dimethyl-3-pentanone & -2.74 & -10.69 & 0.03 & -13.35 & 0.43 & -4.57 & -4.58 \\
\hline Acetophenone & -4.58 & -10.46 & 0.14 & -13.33 & 0.43 & -4.47 & -4.44 \\
\hline
\end{tabular}




\begin{tabular}{|c|c|c|c|c|c|c|c|}
\hline Acetaldehyde & -3.50 & -8.98 & 0.00 & -5.14 & 0.44 & -3.95 & -3.86 \\
\hline Propanal & -3.44 & -9.14 & 0.24 & -7.12 & 0.41 & -3.61 & -3.82 \\
\hline Butanal & -3.18 & -9.01 & -0.28 & -8.91 & 0.44 & -4.06 & -3.99 \\
\hline Pentanal & -3.03 & -8.91 & -0.15 & -10.67 & 0.42 & -3.85 & -3.89 \\
\hline Hexanal & -2.81 & -8.95 & -0.41 & -12.34 & 0.42 & -3.94 & -4.02 \\
\hline Trans-2-Butenal & -4.23 & -8.52 & 0.02 & -9.03 & 0.43 & -3.64 & -3.66 \\
\hline trans,trans-2,4-Hexadienal & -4.63 & -9.21 & -0.01 & -12.28 & 0.43 & -3.97 & -3.96 \\
\hline Benzaldehyde & -4.02 & -9.68 & 0.07 & -12.02 & 0.44 & -4.20 & -4.13 \\
\hline Ethanal & -3.50 & -9.08 & 0.00 & -5.09 & 0.44 & -3.98 & -3.90 \\
\hline Acetic acid & -6.70 & -17.68 & 0.32 & -4.29 & 0.39 & -6.85 & -6.88 \\
\hline Propionic acid & -6.48 & -17.95 & 0.24 & -6.05 & 0.39 & -6.91 & -7.02 \\
\hline Butyric & -6.36 & -17.84 & -0.34 & -7.75 & 0.40 & -7.35 & -7.21 \\
\hline Methyl acetate & -3.32 & -7.18 & 0.04 & -7.98 & 0.43 & -3.06 & -3.07 \\
\hline Ethylacetate & -3.10 & -7.08 & -0.16 & -9.99 & 0.42 & -3.03 & -3.11 \\
\hline n-Propyl acetate & -2.86 & -7.06 & -0.43 & -11.78 & 0.43 & -3.24 & -3.22 \\
\hline Isopropyl acetate & -2.65 & -7.16 & 0.03 & -11.77 & 0.42 & -2.97 & -3.06 \\
\hline Methyl propanoate & -2.93 & -7.48 & 0.05 & -9.86 & 0.42 & -3.09 & -3.19 \\
\hline Ethyl propanoate & -2.80 & -7.14 & -0.11 & -11.88 & 0.42 & -3.06 & -3.12 \\
\hline n-Propyl propanoate & -2.45 & -7.11 & -0.35 & -13.63 & 0.43 & -3.21 & -3.21 \\
\hline Isopropyl propanoate & -2.22 & -7.20 & 0.02 & -13.45 & 0.41 & -2.96 & -3.09 \\
\hline Methyl butanoate & -2.83 & -7.20 & -0.46 & -11.61 & 0.44 & -3.40 & -3.29 \\
\hline Ethyl butanoate & -2.50 & -7.15 & -0.04 & -13.57 & 0.41 & -2.93 & -3.09 \\
\hline n-Propyl butanoate & -2.28 & -6.96 & -0.69 & -15.16 & 0.38 & -2.93 & -3.29 \\
\hline Methyl pentanoate & -2.57 & -7.40 & 0.10 & -13.23 & 0.43 & -3.14 & -3.14 \\
\hline Ethyl pentanoate & -2.52 & -7.15 & -0.22 & -15.26 & 0.41 & -3.05 & -3.17 \\
\hline Methyl benzoate & -4.28 & -8.57 & 0.11 & -14.73 & 0.41 & -3.49 & -3.64 \\
\hline Ethyl formate & -2.65 & -6.79 & -0.52 & -8.49 & 0.45 & -3.31 & -3.14 \\
\hline Methyl amine & -4.56 & -14.20 & 0.11 & -1.50 & 0.38 & -5.40 & -5.49 \\
\hline Ethyl amine & -4.50 & -14.50 & -0.26 & -3.73 & 0.38 & -5.65 & -5.75 \\
\hline n-Propyl amine & -4.39 & -13.76 & -1.02 & -5.66 & 0.40 & -5.94 & -5.76 \\
\hline n-Butyl amine & -4.29 & -14.25 & -0.19 & -7.31 & 0.40 & -5.75 & -5.63 \\
\hline n-Pentyl amine & -4.10 & -14.00 & -0.12 & -9.16 & 0.39 & -5.46 & -5.50 \\
\hline n-Hexyl amine & -4.03 & -13.88 & 0.06 & -10.91 & 0.40 & -5.58 & -5.39 \\
\hline Dimethyl amine & -4.29 & -12.96 & 0.12 & -3.88 & 0.39 & -4.95 & -5.01 \\
\hline Diethyl amine & -4.07 & -12.80 & 0.23 & -8.26 & 0.40 & -5.06 & -4.90 \\
\hline Di-n-propyl amine & -3.66 & -11.99 & -0.54 & -11.94 & 0.37 & -4.68 & -4.88 \\
\hline Di-n-butyl amine & -3.33 & -12.36 & -0.53 & -15.43 & 0.39 & -5.07 & -5.02 \\
\hline Trimethyl amine & -3.24 & -10.13 & 0.05 & -6.35 & 0.42 & -4.21 & -4.33 \\
\hline Triethyl amine & -3.02 & -8.21 & 0.08 & -11.91 & 0.46 & -3.73 & -3.49 \\
\hline Aziridine & -5.42 & -17.41 & 0.04 & -2.81 & 0.38 & -6.66 & -6.77 \\
\hline Azetidine & -5.56 & -14.73 & 0.09 & -5.19 & 0.40 & -5.79 & -5.71 \\
\hline Pyrrolidine & -5.48 & -12.90 & -0.01 & -7.18 & 0.40 & -5.12 & -5.03 \\
\hline Piperidine & -5.11 & -11.28 & 0.07 & -9.00 & 0.40 & -4.44 & -4.37 \\
\hline N-Methylpyrrolidine & -3.98 & -10.37 & 0.12 & -9.49 & 0.43 & -4.36 & -4.41 \\
\hline N-Methylpiperidine & -3.89 & -8.46 & 0.03 & -10.95 & 0.42 & -3.54 & -3.62 \\
\hline Ammonia & -4.31 & -15.97 & 0.00 & 1.25 & 0.37 & -5.93 & -6.23 \\
\hline Aniline & -4.90 & -15.80 & -0.08 & -8.86 & 0.39 & -6.27 & -6.19 \\
\hline Acetamide & -9.71 & -24.34 & 0.27 & -4.58 & 0.41 & -9.83 & -9.75 \\
\hline Propionamide & -9.41 & -24.81 & 0.20 & -6.25 & 0.40 & -9.82 & -9.97 \\
\hline N-Methylacetamide & -10.08 & -18.56 & 0.12 & -8.13 & 0.42 & -7.77 & -7.93 \\
\hline N-Methyl formamide & -10.00 & -17.80 & 0.08 & -6.21 & 0.42 & -7.50 & -7.62 \\
\hline N,N-Dimethyl-acetamide & -8.50 & -12.32 & 0.00 & -10.78 & 0.43 & -5.27 & -5.30 \\
\hline Acetonitrile & -3.89 & -15.15 & 0.00 & -3.36 & 0.45 & -6.77 & -6.51 \\
\hline Propiononitrile & -3.85 & -14.74 & 0.01 & -5.50 & 0.43 & -6.36 & -6.33 \\
\hline Butyronitrile & -3.64 & -14.53 & -0.42 & -7.31 & 0.44 & -6.65 & -6.43 \\
\hline Nitroethane & -3.71 & -9.89 & -0.01 & -7.91 & 0.47 & -4.70 & -4.25 \\
\hline 1-Nitropropane & -3.34 & -9.96 & -0.06 & -9.63 & 0.47 & -4.71 & -4.30 \\
\hline 2-Nitropropane & -3.14 & -9.91 & -0.11 & -11.32 & 0.47 & -4.68 & -4.30 \\
\hline
\end{tabular}




\begin{tabular}{|c|c|c|c|c|c|c|c|}
\hline Nitrobenzene & -4.12 & -6.79 & 0.17 & -13.18 & 0.44 & -2.94 & -2.84 \\
\hline Pyridine & -4.70 & -9.35 & 0.27 & -8.96 & 0.38 & -3.41 & -3.90 \\
\hline 2-Methylpyridine & -4.63 & -10.46 & 0.00 & -10.63 & 0.39 & -4.12 & -4.50 \\
\hline 3-Methylpyridine & -4.77 & -10.28 & -0.02 & -10.64 & 0.41 & -4.22 & -4.43 \\
\hline 2-Etylypyridine & -4.33 & -10.22 & -0.01 & -12.26 & 0.40 & -4.13 & -4.40 \\
\hline 3-Ethylpyridine & -4.60 & -10.15 & 0.04 & -12.26 & 0.41 & -4.12 & -4.35 \\
\hline 2,3-Dimethylpyridine & -4.83 & -11.43 & 0.00 & -12.23 & 0.40 & -4.54 & -4.91 \\
\hline 3,5-Dimethylpyridine & -4.84 & -11.19 & -0.06 & -15.20 & 0.42 & -4.67 & -4.84 \\
\hline 2,6-Dimethylpyridine & -4.60 & -11.19 & 0.03 & -12.30 & 0.41 & -4.56 & -4.80 \\
\hline 2,6-Di-t-butylpyridine & -0.41 & -8.33 & 0.05 & -21.25 & 0.40 & -3.34 & -3.56 \\
\hline N-Methyl-2-pyridone & -10.00 & -15.73 & 0.11 & -12.23 & 0.43 & -6.79 & -6.72 \\
\hline Methanethiol & -1.24 & -4.42 & 0.01 & -4.71 & 0.46 & -2.02 & -1.89 \\
\hline Ethanethiol & -1.30 & -4.21 & 0.10 & -6.72 & 0.44 & -1.83 & -1.77 \\
\hline Benzenethiol & -2.55 & -4.80 & -0.09 & -11.39 & 0.46 & -2.23 & -2.10 \\
\hline Dimethyl sulfide & -1.54 & -9.07 & 0.03 & -6.53 & 0.43 & -3.87 & -3.89 \\
\hline Diethyl sulfide & -1.43 & -8.84 & 0.02 & -10.23 & 0.43 & -3.79 & -3.79 \\
\hline Methyl phenyl sulfide & -2.73 & -7.00 & 0.12 & -13.22 & 0.46 & -3.14 & -2.96 \\
\hline n-Butyramide & NA & -24.76 & -0.07 & -7.89 & 0.41 & -10.17 & -10.05 \\
\hline Isobutyramide & NA & -25.00 & 0.12 & -7.70 & 0.41 & -10.09 & -10.07 \\
\hline Trimethylacetamide & NA & -23.19 & 0.30 & -9.49 & 0.40 & -9.22 & -9.27 \\
\hline 3-Methylbutanamaide & NA & -24.71 & 0.23 & -9.48 & 0.40 & -9.75 & -9.91 \\
\hline 3,3-Dimethylbutanamide & NA & -24.78 & 0.26 & -10.81 & 0.40 & -9.82 & -9.93 \\
\hline N-Ethylacetamide & NA & -18.32 & 0.04 & -9.92 & 0.43 & -7.84 & -7.86 \\
\hline N-Isopropylacetamide & NA & -18.06 & -0.54 & -11.57 & 0.44 & -8.14 & -8.00 \\
\hline N-Tertbutylacetamide & NA & -17.88 & 0.07 & -12.88 & 0.42 & -7.56 & -7.65 \\
\hline N-Ethylpropionamide & NA & -18.52 & -0.06 & -11.71 & 0.44 & -8.09 & -7.99 \\
\hline N-Ethylisobutyramide & NA & -18.85 & -0.14 & -12.94 & 0.43 & -8.21 & -8.17 \\
\hline N-Ethyltrimethylacetamide & NA & -16.83 & 0.05 & -14.44 & 0.44 & -7.42 & -7.21 \\
\hline N-Tertbutyltrimethylacetamide & NA & -15.80 & 0.15 & -17.16 & 0.43 & -6.77 & -6.73 \\
\hline N-Ethyl-N-methyl-acetamide & NA & -12.54 & 0.05 & -12.31 & 0.42 & -5.29 & -5.37 \\
\hline N-Ethyl-N-methyl-propionamide & NA & -12.73 & 0.07 & -13.91 & 0.43 & -5.42 & -5.44 \\
\hline N,N-Diethyl-propionamide & NA & -12.53 & 0.04 & -15.28 & 0.43 & -5.32 & -5.37 \\
\hline $\begin{array}{l}\text { N-Isopropyl-N-tertbutyl-2-methyl- } \\
\text { butanamide }\end{array}$ & NA & -12.04 & 0.68 & -20.97 & 0.40 & -4.59 & -4.88 \\
\hline Methylammonium & -71.30 & -137.37 & 0.01 & 3.58 & 0.51 & -70.02 & -71.43 \\
\hline Ethylammonium & -68.40 & -135.89 & 0.02 & 1.72 & 0.51 & -69.22 & -70.65 \\
\hline Annilinium & -66.00 & -134.43 & 0.14 & -2.82 & 0.51 & -68.46 & -69.83 \\
\hline Dimethylammonium & -63.90 & -117.60 & 0.07 & -1.53 & 0.52 & -61.35 & -61.11 \\
\hline Diethylammonium & -58.90 & -113.90 & 0.07 & -5.25 & 0.52 & -59.47 & -59.19 \\
\hline Di-n-propylammonium & -57.70 & -113.76 & 0.84 & -8.93 & 0.52 & -58.75 & -58.72 \\
\hline Trimethylammonium & -56.60 & -100.52 & 0.09 & -5.85 & 0.54 & -53.89 & -52.22 \\
\hline Triethylammonium & -50.20 & -94.52 & -0.05 & -10.78 & 0.54 & -50.87 & -49.18 \\
\hline N-Methylpiperidine & NA & -96.69 & 0.08 & -10.10 & 0.54 & -52.10 & -50.23 \\
\hline Acetate & -79.90 & -160.89 & 0.13 & 3.76 & 0.45 & -71.55 & -71.52 \\
\hline Propionate & -79.10 & -160.40 & 0.23 & 1.77 & 0.45 & -71.59 & -71.26 \\
\hline Benzoate & -76.00 & -156.03 & 0.46 & -3.45 & 0.44 & -68.84 & -69.21 \\
\hline
\end{tabular}

${ }^{\mathrm{a}} \mathrm{kcal} / \mathrm{mol} .{ }^{\mathrm{b}}$ experimental values are from ref. 43 as compiled by ref. 63. ${ }^{\mathrm{c}}$ Average uncertainties for nonionic and ionic molecules are 0.03 and $0.11 \mathrm{kcal} / \mathrm{mol}$ respectively. ${ }^{\mathrm{d}}$ Average uncertainties for nonionic and ionic molecules are 0.11 and $0.02 \mathrm{kcal} / \mathrm{mol}$ respectively in the gas phase and 0.03 and 0.02 $\mathrm{kcal} / \mathrm{mol}$ in the aqueous phase. ${ }^{\mathrm{e}}$ Average uncertainties for nonionic and ionic molecules are 0.04 and $0.03 \mathrm{kcal} / \mathrm{mol}$ respectively. ${ }^{\mathrm{f}}$ Average uncertainties for nonionic and ionic molecules are 0.00 and 0.00 $\mathrm{kcal} / \mathrm{mol}$ respectively in the gas phase and 0.00 and $0.01 \mathrm{kcal} / \mathrm{mol}$ in the aqueous phase. 
Table 2. The electrostatic solute energies $\left(\left\langle U_{r-s}^{e l}\right\rangle_{B}^{w}\right.$ and $\left.\Delta\left\langle U_{r-r}^{e l}\right\rangle_{B}\right)$ and electrostatic contribution to the free energies of hydration from FEP calculations $\left(\Delta F_{\text {sol }}^{e l}(F E P)\right)$ and model $\mathrm{E}\left(\Delta F_{\text {sol }}^{\text {el }}(\right.$ Model $\left.E)\right)$ for each of the molecules in test set. ${ }^{\mathrm{a}}$ The test set molecules are written as $\mathrm{X} \_\mathrm{Y}$, where $\mathrm{X}$ and $\mathrm{Y}$ are the groups from Figure 3.

\begin{tabular}{|c|c|c|c|c|}
\hline Solute & $\left\langle U_{r-s}^{e l}\right\rangle_{B}^{w} \mathrm{~b}$ & $\Delta\left\langle U_{r-r}^{e l}\right\rangle_{B}{ }^{\mathrm{c}}$ & $\Delta F_{\text {sol }}^{e l}(F E P)^{\mathrm{d}}$ & $\Delta F_{\text {sol }}^{e l}($ Model $E)$ \\
\hline AD1_AD1 & -48.39 & 6.19 & -17.82 & -17.14 \\
\hline AD1_AD2 & -43.58 & 4.05 & -16.40 & -16.53 \\
\hline AD1_AD3 & -36.12 & 0.97 & -13.93 & -14.69 \\
\hline AD1_AL & -41.13 & 1.19 & -15.69 & -15.56 \\
\hline AD1_AN1 & -38.07 & 1.07 & -14.95 & -14.76 \\
\hline AD1_AN2 & -36.50 & 1.07 & -14.29 & -14.13 \\
\hline AD1_AN3 & -33.18 & 0.13 & -13.30 & -13.43 \\
\hline AD1_CA & -52.87 & 13.09 & -16.00 & -15.86 \\
\hline AD1_ES & -31.88 & 3.29 & -12.23 & -11.95 \\
\hline AD1_ET & -30.98 & 1.98 & -11.90 & -11.56 \\
\hline AD1_KA & -34.87 & 1.86 & -13.30 & -13.80 \\
\hline AD1_NL & -37.87 & 1.38 & -14.85 & -15.25 \\
\hline AD1_NO & -34.40 & 1.00 & -14.00 & -14.59 \\
\hline AD1_SU & -32.23 & 0.68 & -12.84 & -13.19 \\
\hline AD1_TH & -28.65 & 0.00 & -11.75 & -11.98 \\
\hline AD2_AD1 & -41.99 & 3.52 & -15.57 & -16.08 \\
\hline AD2_AD2 & -37.12 & 2.47 & -13.76 & -14.90 \\
\hline AD2_AD3 & -27.99 & 0.80 & -11.60 & -11.69 \\
\hline AD2_AL & -34.61 & 1.43 & -13.12 & -13.32 \\
\hline AD2_AN1 & -31.46 & 0.07 & -12.74 & -12.90 \\
\hline AD2_AN2 & -29.99 & 0.02 & -12.54 & -12.31 \\
\hline AD2_AN3 & -26.50 & -0.37 & -11.23 & -11.23 \\
\hline AD2_CA & -44.55 & 9.50 & -15.08 & -14.40 \\
\hline AD2_ES & -25.21 & 1.03 & -10.17 & -10.40 \\
\hline AD2_ET & -23.13 & 0.09 & -9.72 & -9.46 \\
\hline AD2_KA & -27.14 & 0.77 & -11.35 & -11.34 \\
\hline AD2_NL & -31.81 & 1.53 & -12.76 & -13.02 \\
\hline AD2_NO & -27.44 & 0.35 & -11.85 & -12.15 \\
\hline AD2_SU & -25.62 & 1.29 & -10.80 & -10.46 \\
\hline AD2_TH & -22.39 & -0.26 & -9.48 & -9.74 \\
\hline AD3_AD1 & -35.50 & 1.17 & -13.26 & -14.35 \\
\hline AD3_AD2 & -30.88 & 1.44 & -11.59 & -12.66 \\
\hline AD3_AD3 & -22.21 & 0.20 & -9.03 & -9.46 \\
\hline AD3_AL & -27.56 & 0.64 & -10.99 & -10.81 \\
\hline AD3_AN1 & -25.22 & -0.67 & -11.01 & -10.63 \\
\hline AD3_AN2 & -23.02 & 0.63 & -9.71 & -9.20 \\
\hline AD3_AN3 & -20.72 & -0.88 & -9.64 & -9.03 \\
\hline AD3_CA & -38.24 & 8.87 & -12.66 & -12.07 \\
\hline AD3_ES & -17.06 & -0.30 & -7.35 & -7.46 \\
\hline AD3_ET & -16.24 & -0.30 & -6.59 & -6.79 \\
\hline AD3_KA & -21.63 & 0.54 & -8.54 & -9.07 \\
\hline AD3_NL & -25.26 & 0.75 & -10.08 & -10.54 \\
\hline AD3_NO & -22.08 & 0.81 & -9.14 & -9.54 \\
\hline AD3_SU & -17.91 & -0.35 & -7.85 & -7.85 \\
\hline AD3_TH & -15.61 & -0.22 & -7.21 & -6.80 \\
\hline AL_AD1 & -41.84 & 4.41 & -15.49 & -14.58 \\
\hline AL_AD2 & -34.74 & 0.53 & -13.60 & $\begin{array}{l}-13.73 \\
\end{array}$ \\
\hline AL_AD3 & -29.14 & 1.36 & -11.47 & -11.15 \\
\hline AL_AL & -33.46 & 1.06 & -11.89 & -12.08 \\
\hline AL_AN1 & -30.51 & 0.65 & -11.44 & -11.41 \\
\hline
\end{tabular}




\begin{tabular}{|c|c|c|c|c|}
\hline AL_AN2 & -28.86 & 0.27 & -10.64 & -10.93 \\
\hline AL_AN3 & -26.31 & 0.63 & -10.04 & -10.01 \\
\hline AL_CA & -44.66 & 2.83 & -16.58 & -15.99 \\
\hline AL_ES & -25.62 & 1.80 & -9.25 & -9.56 \\
\hline AL_ET & -23.53 & 0.54 & -8.48 & -8.79 \\
\hline AL_KA & -27.09 & 0.59 & -9.80 & -10.64 \\
\hline AL_NL & -31.61 & 1.76 & -12.14 & -11.98 \\
\hline AL_NO & -27.32 & 0.69 & -9.99 & -11.19 \\
\hline AL_SU & -25.53 & 0.61 & -9.47 & -10.00 \\
\hline AL_TH & -20.96 & 0.12 & -7.99 & -8.36 \\
\hline AN1_AD1 & -37.39 & -0.90 & -15.61 & -15.27 \\
\hline AN1_AD2 & -32.05 & 0.69 & -13.05 & -12.88 \\
\hline AN1_AD3 & -25.70 & 0.74 & -10.13 & -10.25 \\
\hline AN1_AL & -28.85 & 0.78 & -10.37 & -10.73 \\
\hline AN1_AN1 & -25.91 & -0.85 & -10.99 & -10.48 \\
\hline AN1_AN2 & -24.66 & 0.56 & -9.71 & -9.43 \\
\hline AN1_AN3 & -21.67 & 0.17 & -8.67 & -8.58 \\
\hline AN1_CA & -40.35 & 0.43 & -15.74 & -15.63 \\
\hline AN1_ES & -21.89 & -0.04 & -8.31 & -9.01 \\
\hline AN1_ET & -20.03 & -0.13 & -7.82 & -7.89 \\
\hline AN1_KA & -23.79 & 0.38 & -9.32 & -9.61 \\
\hline AN1_NL & -27.25 & 0.33 & -11.06 & -11.06 \\
\hline AN1_NO & -22.66 & 0.66 & -9.29 & -9.45 \\
\hline AN1_SU & -21.58 & -0.12 & -8.96 & -8.91 \\
\hline AN1_TH & -16.99 & -0.77 & -7.08 & -7.30 \\
\hline AN2_AD1 & -36.40 & 0.60 & -14.33 & -14.28 \\
\hline AN2_AD2 & -29.69 & 1.01 & -12.01 & -11.78 \\
\hline AN2_AD3 & -23.86 & 0.68 & -9.82 & -9.52 \\
\hline AN2_AL & -27.15 & 0.59 & -10.06 & -10.15 \\
\hline AN2_AN1 & -24.41 & 0.08 & -9.00 & -9.53 \\
\hline AN2_AN2 & -23.01 & 0.01 & -9.22 & -9.00 \\
\hline AN2_AN3 & -19.72 & 0.64 & -7.44 & -7.61 \\
\hline AN2_CA & -38.01 & 6.31 & -14.52 & -12.41 \\
\hline AN2_ES & -20.11 & 1.65 & -6.70 & -7.58 \\
\hline AN2_ET & -17.43 & -0.54 & -7.88 & -7.03 \\
\hline AN2_KA & -21.81 & 0.96 & -8.73 & -8.56 \\
\hline AN2_NL & -25.78 & 1.41 & -10.08 & -10.01 \\
\hline AN2_NO & -21.19 & 0.69 & -8.54 & -8.80 \\
\hline AN2_SU & -19.83 & 0.16 & -8.17 & -8.08 \\
\hline AN2_TH & -14.91 & -0.82 & -6.73 & -6.46 \\
\hline AN3_AD1 & -32.65 & 0.02 & -13.16 & -13.25 \\
\hline AN3_AD2 & -26.77 & 0.24 & -11.24 & -11.09 \\
\hline AN3_AD3 & -20.57 & 0.81 & -8.54 & -8.26 \\
\hline AN3_AL & -24.69 & 0.58 & $\begin{array}{l}-9.33 \\
\end{array}$ & -9.39 \\
\hline AN3_AN1 & -21.89 & 1.40 & -8.25 & -8.17 \\
\hline AN3_AN2 & -19.91 & 0.49 & -8.01 & -7.74 \\
\hline AN3_AN3 & -17.09 & 0.24 & -6.89 & -6.84 \\
\hline AN3_CA & -35.52 & 1.31 & -13.85 & -13.65 \\
\hline AN3_ES & -14.83 & -1.05 & -6.24 & -6.63 \\
\hline AN3_ET & -14.53 & 0.50 & -5.80 & -5.60 \\
\hline AN3_KA & -18.41 & -0.10 & -7.91 & -7.74 \\
\hline AN3_NL & -22.65 & -0.05 & -9.62 & -9.49 \\
\hline AN3_NO & -18.15 & 0.61 & -7.38 & -7.66 \\
\hline AN3_SU & -16.67 & 0.04 & -7.10 & -6.95 \\
\hline AN3_TH & -12.20 & -0.19 & -5.24 & -5.18 \\
\hline CA_AD1 & -41.70 & 0.91 & -16.22 & -16.27 \\
\hline CA_AD2 & -35.79 & 0.72 & -14.37 & -14.41 \\
\hline CA_AD3 & -29.53 & 0.49 & -11.59 & -11.92 \\
\hline CA_AL & -33.95 & 0.70 & -12.22 & -12.71 \\
\hline
\end{tabular}




\begin{tabular}{|c|c|c|c|c|}
\hline CA_AN1 & -31.33 & 1.14 & -12.02 & -11.82 \\
\hline CA_AN2 & -29.28 & 0.15 & -11.29 & -11.41 \\
\hline CA_AN3 & -26.32 & 0.48 & -10.20 & -10.30 \\
\hline CA_CA & -44.75 & 5.61 & -16.41 & -15.32 \\
\hline CA_ES & -25.82 & 1.57 & -9.39 & -9.96 \\
\hline CA_ET & -23.50 & 0.45 & -9.14 & -9.02 \\
\hline CA_KA & -27.73 & 0.61 & -10.88 & -11.14 \\
\hline CA_NL & -30.98 & 0.64 & -12.46 & -12.46 \\
\hline CA_NO & -27.16 & 0.81 & -11.03 & -11.31 \\
\hline CA_SU & -25.03 & 0.74 & -9.69 & -9.98 \\
\hline CA_TH & -21.42 & 0.71 & -8.36 & -8.51 \\
\hline ES_AD1 & -32.53 & 2.61 & -12.15 & -12.51 \\
\hline ES_AD2 & -26.62 & 2.36 & -10.23 & -10.43 \\
\hline ES_AD3 & -19.87 & 2.25 & -7.30 & -7.58 \\
\hline ES_AL & -26.98 & 2.32 & -8.44 & -9.90 \\
\hline ES_AN1 & -20.09 & 0.14 & $\begin{array}{l}-7.89 \\
\end{array}$ & -8.20 \\
\hline ES_AN2 & -18.59 & 0.08 & -7.32 & -7.60 \\
\hline ES_AN3 & $\begin{array}{l}-18.68 \\
\end{array}$ & 1.73 & $\begin{array}{l}-6.55 \\
\end{array}$ & -7.09 \\
\hline ES_CA & -34.14 & 8.28 & $\begin{array}{l}-11.13 \\
\end{array}$ & -10.62 \\
\hline ES_ES & -19.04 & 4.26 & -4.51 & -6.36 \\
\hline ES_ET & -14.92 & 1.74 & -5.13 & -5.41 \\
\hline ES_KA & -18.37 & 0.79 & -7.12 & -7.56 \\
\hline ES_NL & -21.41 & 0.92 & -8.94 & -8.81 \\
\hline ES_NO & -18.35 & 0.85 & -7.41 & -7.85 \\
\hline ES_SU & -14.75 & 0.17 & -6.31 & -6.27 \\
\hline ES_TH & -11.85 & -1.62 & -4.96 & -5.79 \\
\hline ET_AD1 & -31.26 & 3.95 & -11.40 & -10.89 \\
\hline ET_AD2 & -24.86 & 1.38 & -9.84 & -9.64 \\
\hline ET_AD3 & -18.83 & 0.88 & -7.32 & -7.37 \\
\hline ET_AL & -23.02 & 0.81 & -8.29 & -8.49 \\
\hline ET_AN1 & -20.26 & 0.83 & -7.87 & -7.61 \\
\hline ET_AN2 & -18.51 & 0.47 & -7.41 & -7.07 \\
\hline ET_AN3 & -15.59 & 0.27 & -6.47 & -6.11 \\
\hline ET_CA & -33.63 & 4.35 & -12.86 & -11.47 \\
\hline ET_ES & -13.35 & 0.32 & -5.32 & -5.35 \\
\hline ET_ET & -13.30 & 0.21 & -5.07 & -5.13 \\
\hline ET_KA & -16.11 & 1.17 & -6.35 & -6.14 \\
\hline ET_NL & -19.86 & 1.09 & -8.07 & -7.71 \\
\hline ET_NO & -15.37 & -1.04 & -6.92 & -7.04 \\
\hline ET_SU & -15.11 & 0.71 & -5.91 & -5.92 \\
\hline ET_TH & -10.89 & 0.41 & -4.51 & -4.30 \\
\hline KA_AD1 & -34.23 & 1.16 & -13.50 & -13.83 \\
\hline KA_AD2 & -27.61 & 0.54 & -11.39 & -11.64 \\
\hline KA_AD3 & -20.91 & 0.34 & -9.06 & -8.84 \\
\hline KA_AL & -26.34 & 0.90 & -9.80 & -10.21 \\
\hline KA_AN1 & -24.21 & 0.48 & -9.44 & -9.75 \\
\hline KA_AN2 & -22.55 & 1.45 & -8.77 & -8.67 \\
\hline KA_AN3 & -19.55 & 0.71 & -7.65 & -7.88 \\
\hline KA_CA & -38.06 & 7.39 & -13.64 & -12.60 \\
\hline KA_ES & -18.64 & 0.22 & -7.59 & -7.92 \\
\hline KA_ET & -15.28 & -1.28 & -6.72 & -6.80 \\
\hline KA_KA & -20.30 & 0.56 & -7.84 & -8.49 \\
\hline KA_NL & -23.91 & 0.52 & -10.11 & -10.06 \\
\hline KA_NO & -20.71 & 1.38 & -8.49 & -8.67 \\
\hline KA_SU & -18.24 & 0.47 & -8.09 & -7.64 \\
\hline KA_TH & -14.49 & 0.10 & -6.07 & -6.19 \\
\hline NL_AD1 & -37.50 & 0.76 & -14.78 & -15.36 \\
\hline NL_AD2 & -31.62 & 0.94 & -12.91 & -13.19 \\
\hline NL_AD3 & -25.31 & 0.66 & -10.33 & -10.60 \\
\hline
\end{tabular}




\begin{tabular}{|c|c|c|c|c|}
\hline NL_AL & -30.20 & 0.99 & -11.96 & -11.72 \\
\hline NL_AN1 & -28.19 & -0.89 & -11.56 & -11.94 \\
\hline NL_AN2 & -26.41 & 0.01 & -10.93 & -10.84 \\
\hline NL_AN3 & -23.17 & 0.48 & -10.00 & -9.49 \\
\hline NL_CA & -41.86 & 3.66 & -17.15 & -15.69 \\
\hline NL_ES & -22.04 & 0.16 & -9.31 & -9.40 \\
\hline NL_ET & -19.98 & 0.36 & -8.52 & -8.06 \\
\hline NL_KA & -24.30 & 0.80 & -9.74 & -10.11 \\
\hline NL_NL & -29.63 & 0.16 & -12.81 & -12.67 \\
\hline NL_NO & -24.84 & 0.34 & -11.10 & -10.99 \\
\hline NL_SU & -22.32 & 0.27 & -9.86 & -9.48 \\
\hline NL_TH & -18.90 & -0.65 & -8.62 & -8.40 \\
\hline NO_AD1 & -33.93 & 1.56 & -13.64 & -14.14 \\
\hline NO_AD2 & -27.61 & 0.96 & -12.02 & -11.96 \\
\hline NO_AD3 & -21.25 & 0.64 & -9.48 & -9.24 \\
\hline NO_AL & -26.11 & 0.36 & -10.52 & -10.82 \\
\hline NO_AN1 & -22.39 & 0.54 & -8.93 & -9.38 \\
\hline NO_AN2 & -20.76 & 0.78 & -8.68 & -8.58 \\
\hline NO_AN3 & -17.24 & 0.35 & -7.47 & -7.38 \\
\hline NO_CA & -36.66 & 1.85 & -14.32 & -14.95 \\
\hline NO_ES & -16.90 & -0.07 & -6.97 & -7.61 \\
\hline NO_ET & -12.67 & 0.27 & -4.95 & -5.33 \\
\hline NO_KA & -19.75 & 0.83 & -8.75 & -8.49 \\
\hline NO_NL & -26.81 & 0.40 & -11.81 & -11.85 \\
\hline NO_NO & $\begin{array}{l}-18.92 \\
\end{array}$ & 1.00 & -8.21 & -8.37 \\
\hline NO_SU & -17.99 & 3.41 & -6.48 & -6.54 \\
\hline NO_TH & -14.10 & 0.02 & -6.40 & -6.32 \\
\hline SU_AD1 & -32.21 & 0.74 & -12.71 & -13.15 \\
\hline SU_AD2 & -26.67 & 1.66 & -10.72 & -10.75 \\
\hline SU_AD3 & -19.62 & 0.68 & -7.89 & -8.14 \\
\hline SU_AL & -25.36 & 0.83 & -9.26 & -9.85 \\
\hline SU_AN1 & -21.75 & -0.38 & -9.40 & -9.09 \\
\hline SU_AN2 & -20.28 & 0.35 & -8.31 & -8.18 \\
\hline SU_AN3 & -17.27 & 0.13 & -7.23 & -7.16 \\
\hline SU_CA & -35.92 & 4.79 & -13.56 & -12.79 \\
\hline SU_ES & -18.41 & 1.82 & -6.46 & -7.14 \\
\hline SU_ET & -14.61 & 0.17 & -5.96 & -5.93 \\
\hline SU_KA & -18.85 & 1.23 & -7.47 & -7.58 \\
\hline SU_NL & -22.75 & 0.61 & -9.67 & -9.52 \\
\hline SU_NO & -18.58 & 0.01 & -8.19 & -8.33 \\
\hline SU_SU & -16.68 & 0.08 & -7.07 & -7.14 \\
\hline SU_TH & -12.92 & 0.31 & -5.58 & -5.42 \\
\hline TH_AD1 & -27.64 & -0.41 & -11.32 & -11.73 \\
\hline TH_AD2 & -21.61 & 0.50 & -9.47 & -9.08 \\
\hline TH_AD3 & -15.70 & 0.20 & -6.52 & -6.66 \\
\hline TH_AL & -20.36 & 0.47 & -7.61 & -7.98 \\
\hline TH_AN1 & $\begin{array}{l}-17.23 \\
\end{array}$ & -0.28 & $\begin{array}{l}-6.68 \\
\end{array}$ & -7.19 \\
\hline TH_AN2 & -15.53 & -0.30 & $\begin{array}{l}-6.33 \\
\end{array}$ & -6.50 \\
\hline TH_AN3 & -12.92 & 0.20 & -5.26 & -5.32 \\
\hline TH_CA & -30.57 & 0.04 & $\begin{array}{l}-13.01 \\
\end{array}$ & -12.54 \\
\hline TH_ES & $\begin{array}{l}-13.98 \\
\end{array}$ & 2.08 & -4.33 & -5.11 \\
\hline TH_ET & -9.78 & 0.06 & -3.89 & -3.99 \\
\hline TH_KA & -13.21 & 0.17 & -5.97 & -5.61 \\
\hline TH_NL & $\begin{array}{l}-19.67 \\
\end{array}$ & 0.93 & -7.71 & -8.06 \\
\hline TH_NO & -14.18 & 0.65 & -5.90 & -6.07 \\
\hline TH_SU & -11.60 & -0.37 & -5.03 & -5.14 \\
\hline TH_TH & -8.01 & 0.23 & -3.76 & -3.34 \\
\hline AD1_CAT1 & -152.31 & 20.55 & -63.07 & -61.01 \\
\hline AD1_CAT2 & -131.59 & 19.31 & -55.96 & -51.99 \\
\hline
\end{tabular}




\begin{tabular}{|c|c|c|c|c|}
\hline AD1_CAT3 & -115.19 & 12.80 & -52.91 & -47.41 \\
\hline AD1_ANI & -188.63 & 24.98 & -69.56 & -69.63 \\
\hline AD2_CAT1 & -144.97 & 19.35 & -62.20 & -59.67 \\
\hline AD2_CAT2 & -128.34 & 20.56 & -53.77 & -51.20 \\
\hline AD2_CAT3 & -110.27 & 18.91 & -46.90 & -43.40 \\
\hline AD2_ANI & -177.46 & 17.82 & -70.91 & -69.83 \\
\hline AD3_CAT1 & -142.99 & 18.81 & -61.48 & -58.99 \\
\hline AD3_CAT2 & -120.76 & 17.83 & -52.06 & -48.89 \\
\hline AD3_CAT3 & -104.88 & 17.05 & -46.58 & -41.72 \\
\hline AD3_ANI & -177.12 & 7.85 & -74.43 & -74.04 \\
\hline AL_CAT1 & -149.89 & 24.90 & -61.76 & -55.80 \\
\hline AL_CAT2 & -132.81 & 23.28 & -56.24 & -48.90 \\
\hline AL_CAT3 & -113.95 & 12.37 & -52.88 & -45.35 \\
\hline AL_ANI & -173.50 & 12.75 & -72.84 & -65.73 \\
\hline AN1_CAT1 & -146.81 & 12.92 & -63.22 & -61.02 \\
\hline AN1_CAT2 & -127.04 & 12.18 & -57.00 & -52.35 \\
\hline AN1_CAT3 & -109.11 & 10.42 & -54.22 & -44.98 \\
\hline AN1_ANI & -172.09 & 0.56 & -74.93 & -71.73 \\
\hline AN2_CAT1 & -147.16 & 26.29 & -62.76 & -55.09 \\
\hline AN2_CAT2 & -125.79 & 23.00 & -55.81 & -46.85 \\
\hline AN2_CAT3 & -108.23 & 17.72 & -50.71 & -41.25 \\
\hline AN2_ANI & -168.80 & 0.62 & -74.37 & -70.33 \\
\hline AN3_CAT1 & -143.48 & 11.33 & -69.35 & -61.19 \\
\hline AN3_CAT2 & -123.67 & 9.74 & -63.04 & -52.76 \\
\hline AN3_CAT3 & -106.06 & 0.47 & -55.63 & -48.90 \\
\hline AN3_ANI & -166.17 & 0.46 & -73.27 & -70.51 \\
\hline CAT1_AD1 & -151.41 & 20.24 & -62.56 & -60.74 \\
\hline CAT1_AD2 & -145.60 & 19.83 & -62.44 & -59.74 \\
\hline CAT1_AD3 & -140.05 & 16.37 & -61.63 & -58.75 \\
\hline CAT1_AL & -151.43 & 13.45 & -70.38 & -61.60 \\
\hline CAT1_AN1 & -145.93 & 23.62 & -63.28 & -55.74 \\
\hline CAT1_AN2 & -145.21 & 13.51 & -64.17 & -60.03 \\
\hline CAT1_AN3 & -138.39 & 1.53 & -69.71 & -63.38 \\
\hline CAT1_CA1 & -159.12 & 19.30 & -69.82 & -63.73 \\
\hline CAT1_ES & -146.51 & 16.01 & -68.07 & -61.98 \\
\hline CAT1_ET & -130.97 & 5.83 & -65.39 & -57.03 \\
\hline CAT1_KA & -139.82 & 16.09 & -64.17 & -58.77 \\
\hline CAT1_NL & -153.77 & 12.58 & -73.21 & -67.07 \\
\hline CAT1_NO & -145.68 & 14.03 & -67.90 & -64.99 \\
\hline CAT1_SU & -141.90 & 18.14 & -63.29 & -58.79 \\
\hline CAT1_TH & -137.79 & 6.08 & -69.08 & -62.56 \\
\hline CAT2_AD1 & -131.98 & 20.14 & -55.31 & -51.79 \\
\hline CAT2_AD2 & -119.41 & 17.38 & -52.21 & -48.47 \\
\hline CAT2_AD3 & -119.35 & 17.59 & -50.75 & -48.34 \\
\hline CAT2_AL & -127.89 & 19.40 & -56.08 & -48.44 \\
\hline CAT2_AN1 & -123.38 & 22.55 & -53.59 & -45.95 \\
\hline CAT2_AN2 & -122.50 & 22.93 & -52.16 & -45.38 \\
\hline CAT2_AN3 & -116.42 & 8.80 & -55.00 & -49.84 \\
\hline CAT2_CA1 & -135.80 & 17.20 & -59.81 & -54.05 \\
\hline CAT2_ES & -121.21 & 13.98 & -56.69 & -50.93 \\
\hline CAT2_ET & -115.81 & 10.35 & -53.50 & -48.06 \\
\hline CAT2_KA & -118.42 & 15.35 & -52.79 & -48.96 \\
\hline CAT2_NL & -130.01 & 11.35 & -63.10 & -56.36 \\
\hline CAT2_NO & -123.14 & 15.22 & -58.77 & -53.28 \\
\hline CAT2_SU & -120.63 & 18.08 & -52.37 & -48.71 \\
\hline CAT2_TH & -115.74 & 4.82 & -59.51 & -52.69 \\
\hline CAT3_AD1 & -107.88 & 8.10 & -51.09 & -46.21 \\
\hline CAT3_AD2 & -102.85 & 7.69 & -48.70 & -45.20 \\
\hline CAT3_AD3 & -99.04 & 6.99 & -47.60 & -43.73 \\
\hline
\end{tabular}




\begin{tabular}{|c|c|c|c|c|}
\hline CAT3_AL & -105.45 & 4.46 & -52.63 & -45.09 \\
\hline CAT3_AN1 & -102.69 & 4.96 & -50.93 & -44.54 \\
\hline CAT3_AN2 & -101.47 & 4.41 & -50.29 & -44.24 \\
\hline CAT3_AN3 & -95.51 & 4.61 & -50.24 & -42.09 \\
\hline CAT3_CA1 & -116.93 & 6.50 & -56.46 & -50.33 \\
\hline CAT3_ES & -103.11 & 5.67 & -52.45 & -46.28 \\
\hline CAT3_ET & -94.03 & 7.92 & -48.37 & -39.24 \\
\hline CAT3_KA & -97.65 & 6.24 & -48.18 & -43.42 \\
\hline CAT3_NL & -109.58 & 6.14 & -55.49 & -49.13 \\
\hline CAT3_NO & -101.88 & 3.77 & -51.94 & -48.44 \\
\hline CAT3_SU & -97.55 & 6.40 & -49.48 & -43.30 \\
\hline CAT3_TH & -94.89 & 0.05 & -50.14 & -45.05 \\
\hline CA1_CAT1 & -149.43 & 11.47 & -70.21 & -62.88 \\
\hline CA1_CAT2 & -131.57 & 13.33 & -58.05 & -53.89 \\
\hline CA1_CAT3 & -109.62 & 10.30 & -54.45 & -45.26 \\
\hline CA1_ANI & -177.22 & 24.41 & -65.43 & -63.91 \\
\hline ANI_AD1 & -177.54 & 23.19 & -66.38 & -65.68 \\
\hline ANI_AD2 & -172.65 & 17.95 & -67.42 & -67.67 \\
\hline ANI_AD3 & -164.56 & 3.90 & -72.21 & -70.28 \\
\hline ANI_AL & -169.31 & 6.27 & -72.82 & -66.67 \\
\hline ANI_AN1 & -164.90 & 6.61 & -71.68 & -66.20 \\
\hline ANI_AN2 & -166.46 & 7.48 & -73.51 & -66.49 \\
\hline ANI_AN3 & -166.70 & 3.05 & -73.87 & -69.63 \\
\hline ANI_CA1 & -160.38 & 20.13 & -60.82 & -58.65 \\
\hline ANI_ES & -155.41 & 2.14 & -69.19 & -67.05 \\
\hline ANI_ET & -160.33 & 1.74 & -71.37 & -66.32 \\
\hline ANI_KA & -160.97 & 3.60 & -70.50 & -68.84 \\
\hline ANI_NL & -155.65 & 1.05 & -68.91 & -67.63 \\
\hline ANI_NO & -157.25 & 4.33 & -69.03 & -69.75 \\
\hline ANI_SU & -158.77 & 3.23 & -69.04 & -68.04 \\
\hline ANI_TH & -158.35 & 1.97 & -70.40 & -68.41 \\
\hline ES_CAT1 & -148.15 & 15.71 & -66.43 & -62.91 \\
\hline ES_CAT2 & -128.53 & 15.14 & -58.25 & -53.86 \\
\hline ES_CAT3 & -108.32 & 13.80 & -51.31 & -44.89 \\
\hline ES_ANI & -157.08 & 1.35 & -69.66 & -68.12 \\
\hline ET_CAT1 & -130.45 & 11.30 & -60.18 & -54.30 \\
\hline ET_CAT2 & -121.27 & 16.95 & -53.82 & -47.55 \\
\hline ET_CAT3 & -101.96 & 14.75 & -46.73 & -39.75 \\
\hline ET_ANI & -166.28 & 1.40 & -73.10 & -68.95 \\
\hline KA_CAT1 & -142.31 & 16.60 & -64.95 & -59.71 \\
\hline KA_CAT2 & -123.95 & 16.47 & -57.90 & -51.05 \\
\hline KA_CAT3 & -104.28 & 14.37 & -51.35 & -42.70 \\
\hline KA_ANI & -169.21 & 4.75 & -73.46 & -71.94 \\
\hline NL_CAT1 & -156.41 & 14.87 & -73.41 & -67.23 \\
\hline NL_CAT2 & -137.06 & 13.75 & -66.26 & -58.57 \\
\hline NL_CAT3 & -117.09 & 2.73 & -60.43 & -54.32 \\
\hline NL_ANI & -166.08 & 1.72 & -72.60 & -71.90 \\
\hline NO_CAT1 & -152.72 & 13.09 & -70.50 & -68.93 \\
\hline NO_CAT2 & -129.35 & 11.15 & -61.93 & -58.35 \\
\hline NO_CAT3 & -110.10 & 10.08 & -54.43 & -49.38 \\
\hline NO_ANI & -158.06 & 5.80 & -66.20 & -69.45 \\
\hline SU_CAT1 & -142.81 & 17.87 & -63.32 & -59.34 \\
\hline SU_CAT2 & -122.82 & 17.39 & -54.66 & -50.08 \\
\hline SU_CAT3 & -106.15 & 15.83 & -47.46 & -42.90 \\
\hline SU_ANI & -165.34 & 2.32 & -71.92 & -71.31 \\
\hline TH_CAT1 & -139.93 & 6.55 & -68.46 & -63.36 \\
\hline TH_CAT2 & -118.61 & 7.33 & -59.41 & -52.86 \\
\hline TH_CAT3 & -100.15 & 5.10 & -52.15 & -45.15 \\
\hline TH_ANI & -160.99 & 1.44 & -70.77 & -69.79 \\
\hline
\end{tabular}




\begin{tabular}{|l|l|l|l|l|}
\hline CAT1_CAT1 & -320.98 & 2.84 & -162.22 & -165.43 \\
\hline CAT1_CAT2 & -295.76 & 1.01 & -151.43 & -153.27 \\
\hline CAT1_CAT3 & -279.13 & 1.88 & -143.75 & -144.17 \\
\hline CAT2_CAT1 & -293.77 & 0.99 & -151.35 & -152.24 \\
\hline CAT2_CAT2 & -270.72 & 0.80 & -141.29 & -140.36 \\
\hline CAT2_CAT3 & -252.41 & 1.06 & -132.51 & -130.70 \\
\hline CAT3_CAT1 & -274.17 & 2.32 & -140.21 & -141.36 \\
\hline CAT3_CAT2 & -249.38 & 0.89 & -130.05 & -129.21 \\
\hline
\end{tabular}

${ }^{\mathrm{a}}$ all values are $\mathrm{kcal} / \mathrm{mol} .{ }^{\mathrm{b}}$ Average uncertainties for $0, \pm 1, \pm 2$ net charge and zwitterionic molecules are $0.38,1.04,0.79$, and $6.93 \mathrm{kcal} / \mathrm{mol}$ respectively. ${ }^{\mathrm{c}}$ Average uncertainties for $0, \pm 1, \pm 2$ net charge and zwitterionic molecules are $0.35,1.5,0.05$, and $0.22 \mathrm{kcal} / \mathrm{mol}$ respectively in the gas phase and 0.25 , $0.56,0.30$, and $4.00 \mathrm{kcal} / \mathrm{mol}$ in the aqueous phase. ${ }^{\mathrm{d}}$ Average uncertainties for $0, \pm 1, \pm 2$ net charge and zwitterionic molecules are $0.11,0.62,0.32$, and $0.69 \mathrm{kcal} / \mathrm{mol}$ respectively in the gas phase and 0.14 , $0.24,0.26$, and $0.78 \mathrm{kcal} / \mathrm{mol}$ in the aqueous phase. 